\title{
Les crues d'octobre 1960 dans le centre de la France
}

\section{The 1960 floods in Central France}

\author{
PAR J. JACQUET \\ INGĚNIEUR A LA DIVISION HYDROLOGIE \\ DU SERVICE DES ÉTUDES HYDRAULIOUES D'E.D.F.

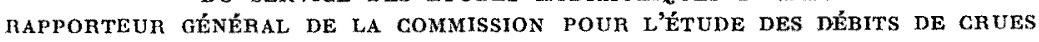

\begin{abstract}
Dans son exposé, l'auteur fait une synthèse des travaux de la Commission pour l'étude des débits de crues lors de sa réunion du 5 mai 1961 , analyse et commente les communications présentées au cours de cette réunion.

Dans la conclusion de cette étude, il propose les voies dans lesquelles il conviendrait de développer les efforts en vue d'apporter une contribution efficace $\dot{a}$ la prévision et à l'annonce des crues et, en matière de protection contre celles$c i$, il énonce les critères à prendre en considération.
\end{abstract}

This paper contains a brief summary of the Commission for the Study of Flood Flows' work during its meeting of the 5th May 1961, with an analysis and comments on the papers presented at the time.

The lines along which the main effort should be made in developing flood-forecasting methods are suggested at the end of the article, also the factors to allow for in taking protective measures.
La Commission pour l'Etude des Débits de Crues, après avoir étudié au cours d'une première série de sessions, de 1951 à 1957 , le problème des crues envisagé principalement sous l'aspect de la détermination des débits maximaux de crues à prévoir pour un barrage, a depuis 1958, sous la présidence de M. l'Inspecteur Général Deymié, élargi ses investigations à l'ensemble des questions se rapportant à l' « hydrométéorologie » des crues, c'est-à-dire à leur formation, leur évolution et leur contrôle. Cette nouvelle orientation ne signifie pas que l'objet premier des travaux de la Commission ait été épuisé : replacé dans un cadre plus vaste, on peut espérer que le problème de la détermination des débits maximaux pour le calcul d'un ouvrage en rivière pourra bénéficier ainsi de nouvelles voies d'approche.

Dans cette perspective, l'étude de phénomènes particuliers, tels que les crues cévenoles de 1958 ou celles de 1960 dans le Centre de la France, s'inscrit naturellement et répond au souci de définir une méthodologie d'étude de ces phénomènes complexes, conjugué à celui du rassemblement d'une documentation de base sur les crues des différents bassins fluviaux français : documentation dont l'utilité se fait de plus en plus sentir pour l'aménagement du territoire. C'est dans cet esprit que les crues d'octobre 1960, affectant les affluents de la Loire et de la Dordogne issus du plateau de Millevaches, ont été mises à l'étude étant donné leur caractère exceptionnel et l'importance de la documentation expérimentale (mesures et observations) réunie à cette occasion. La qualité et l'originalité des rapports présentés à la Commission n'ont pas démenti l'intérêt de ce choix.

Il faut ici insister sur le caractère concerté de la recherche en matière d'hydrométéorologie des crues : une vue compréhensive de ces phénomènes nécessite en effet la collaboration de spécialistes des diverses disciplines ou techniques dont relève leur étude. Du fait de la diversité des organismes auxquels appartiennent ces spécialistes, la Société Hydrotechnique de France constitue le lieu de rencontre tout désigné pour la confrontation, sur le plan scientifique et technique, des résultats de leurs analyses. L'objet de telles confrontations étant de dégager des conclusions de portée générale ou tout au moins des orienta- 
tions de recherches concernant les trois problèmes fondamentaux posés par le contrôle des phénomènes naturels :

$1^{\circ}$ La prévision proprement dite qui ressort principalement à la Météorologie;

$2^{\circ}$ L'annonce qui doit permettre de suivre l'évolution de la crue dans le temps et l'espace et fait appel pour cela aux techniques de l'Hydrologie (relations pluie-débit) et de l'Hydraulique fluviale (propagation et stockage des crues);

$3^{\circ}$ La protection contre les inondations et leurs effets dans des zones critiques définies d'après des critères géomorphologiques et économiques. Ici devront intervenir les ressources du calcul des probabilités : il s'agit de s'assurer contre un risque déterminé par l'importance du phénomène à craindre (débit ou volume d'eau maximum) et par sa fréquence ou probabilité d'occurrence.

C'est en nous référant à ces trois aspects de la question que nous allons examiner successivement les mémoires présentés le 5 mai dernier.

\section{Localisation du phénomène étudié}

Le phénomène étudié intéresse une zone de 5 à $6000 \mathrm{~km}^{2}$ de forme grossièrement elliptique, centrée sur le Plateau de Millevaches, dont le grand axe $(150 \mathrm{~km})$ orienté SSW-NNE va de Brive sur la Corrèze à Teillet-Argenty sur le Cher, et le petit axe $(50 \mathrm{~km})$ d'Eymoutiers sur la Vienne au Pont du Loubeix sur la Diège.

Cette zone cö̈ncide à peu près avec celle couverte par l'isohyète $140 \mathrm{~mm}$ des précipitations reçues entre le 3 octobre 1960 à $6 \mathrm{~h}$ et le 4 en fin de matinée, le paroxysme de la pluie ayant été atteint dans la soirée du 3 sur le Plateau de Millevaches.

Ces précipitations, extraordinaires pour la région, vont provoquer des crues extrêmement brutales dont les maximums se produisent entre $1 \mathrm{~h}$ et $7 \mathrm{~h}$ du matin le 4 octobre aux stations de jaugeage situées à l'intérieur de la zone étudiée sur les cours d'eau affluents de la Loire tels que la Vienne - et ses affluents Maulde, Taurion, Creuse - le Cher et ses affluents Tardes et Vouèze, ou affluents de la Dordogne: Diège, Luzège, Doustre, Vézère et Corrèze

\section{A. - PREVISION DES CRUES}

Par «prévision» il faut entendre ici celle des fortes précipitations à l'origine des crues, réservant à l' «annonce des crues» le soin d'opérer la transformation en débits des informations sur la pluie reçue par un bassin versant et de prévoir l'évolution dans le temps de l'hydrogramme ainsi déterminé.

Quels enseignements peut-on tirer de l'analyse de la situation météorologique qui a provoqué les pluies des 3 et 4 octobre 1960 : peut-on en déduire des critères de prévision efficaces et peuton raisonnablement espérer localiser à l'avance dans l'espace et dans le temps et chiffrer quantitativement de telles précipitations?

A ces questions, M. Fontaine apporte les réponses suivantes :

$1^{\circ}$ La situation météorologique à l'origine de la crue du 4 octobre dans le nord-ouest du Massif Central est un cas «hors série» de fréquence rare;

$2^{\circ}$ On peut définir des situations météorologiques types susceptibles d'engendrer des crues importantes dans cette région;

$3^{\circ}$ La localisation des maximums pluviométriques et la prévision quantitative des précipitations sont des problèmes dont la résolution n'est pas encore acquise, mais des méthodes analogiques permettent de fournir actuellement des éléments de prévision utilisables.

I-Phénomène remarquable et assez inattendu : la coïncidence fortuite de date des crues cévenoles (4 octobre 1958) et du Massif Central (4 octobre 1960) se double d'une analogie réelle des situations météorologiques qui se rattachent à un même type de temps (circulation méridienne $\dot{a}$ composante sud) étudié par M. Fontaine pour expliquer les averses extraordinaires dont le S.-E. des Cévennes a été le théâtre en 1958. Ce type de temps est caractérisé par la présence d'une dépression au voisinage de l'Irlande, se prolongeant par un thalweg en direction du Portugal, jointe à celle d'un anticyclone sur l'Europe Centrale et les Balkans. A ces centres d'action sont liées des masses d'air froid (polaire maritime circulant autour de la dépression et continental autour de l'anticyclone) dont le déplacement relatif amène la constitution d'une zone de blocage freinant le déplacement vers l'est de la perturbation correspondant à l'advection d'air chaud et humide (tropical maritime) entre les deux courants froids. Si l'occlusion de la perturbation ne se produit pas (par exemple affaiblissement du courant froid polaire maritime, postérieur à la perturbation), le conflit entre les masses d'air (polaire et tropical mari- 
time) engendre des précipitations extrêmement violentes. Le problème est alors de savoir localiser le phénomène pour émettre une prévision efficace:

Le 3 octobre 1960 , on était en droit de prévoir à très brève échéance un maximum pluviométrique Cévenol alors qu'il s'est produit au nordouest du Massif Central. Une comparaison plus poussée des situations météorologiques de 1958 à 1960, apparemment analogues, fournit, par l'analyse des masses d'air en présence, un moyen de différencier à temps ces situations. Deux circonstances ont joué en effet simultanément en 1960 pour empêcher le franchissement du Massif Central par l'air chaud et humide dont le courant aurait dû normalement se déplacer rapidement vers l'est : la masse d'air froid continental était plus épaisse et plus avancée vers l'ouest et la masse d'air polaire maritime plus faible qu'en 1958. Ainsi bloqué à l'est par l'air continental et comprimé à l'ouest par l'air froid d'origine atlantique, l'air chaud et humide progresse vers le nord et une zone frontale de conflit s'établit dans l'après-midi du 3 octobre 1960, engendrant un premier maximum pluviométrique sur l'Isle, la Vézère, la Corrèze, la Creuse et le Cher. Puis une nouvelle advection d'air chaud méditerranéen est violemment rejetée dans la soirée du 3 contre le Plateau de Millevaches, qui se trouve alors surmonté d'un dôme d'air froid accentuant le relief du plateau pour soulever la masse d'air chaud, d'où résulte le regain d'intensité des précipitations se propageant d'ouest en est tel qu'il apparaît sur les pluviogrammes des stations de la Corrèze au Cher entre $21 \mathrm{~h}$ le 3 et $3 \mathrm{~h}$ le 4 octobre. Ainsi, c'est à la succession de deux conflits de masses d'air à moins de $12 \mathrm{~h}$ d'intervalle qu'est due l'exceptionnelle, importance des précipitations recueillies sur le Plateau de Millevaches. Une question vient alors $\dot{a}$ l'esprit: quelle est la fréquence d'occurrence d'une situation de ce type sur la zone étudiée?

II - Pour répondre à cette question, M. Fontaine a analysé les situations météorologiques à l'origine des crues importantes de la Corrèze et de la Creuse au cours des 40 dernières années. Il en est résulté la définition de 6 situations-types pour lesquelles des fiches «signalétiques» ont été établies comportant, en plus des schémas relatifs aux champs de pression, fronts et masses d'air, des données détaillées et chiffrées sur les types de temps, perturbations, précipitations et crues associées à ces situations.

Il faut insister ici sur l'importance, pour le progrès de l'hydrométéorologie, de la constitution d'un tel fichier systématique des situations météorologiques génératrices de crues : des analyses de ce genre permettent de préciser les critères à mettre en œuvre dans la prévision des crues. A la lumière de ces résultats, le caractère pluvial océanique de la plupart des crues de la Creuse et de la Corrèze apparaît nettement, liée à la fréquence et l'espacement dans le temps du passage des familles de perturbations venant de l'Océan. Mais une influence méditerranéenne est également indéniable pour les crues de printemps et d'automne dont les situations-types sont caractérisées par des courants méridiens à composante sud et par une grande instabilité des masses d'air. Ce phénomène vaut d'être noté sur des bassins dont la position est excentrique par rapport aux zones habituellement soumises à ces influences.

On pouvait penser que cette dernière circonstance permettrait de rattacher la situation du 3 octobre 1960 à l'une de ces situations-types. Or il est apparu que, si la situation en question appartient à la catégorie des circulations méridiennes à composante sud, le rapprochement de ses éléments constitutifs tant en surface qu'en altitude avec les situations-types de cette catégorie est absolument impossible. Par contre, elle s'identifie remarquablement à l'un des types de situations responsables des plus importantes crues cévenoles!'Il ne s'agit donc pas d'exacerbation d'une situation « classique» mais d'un événement «hors série»de fréquence très faible dont la réalisation nécessite la réunion d'un nombre élevé de facteurs.

III - Dans ces conditions, peut-on localiser à l'avance l'épicentre des précipitations et leur importance quantitative?

Même en limitant l'échéance de la prévision à 12 ou $24 \mathrm{~h}$, l'étude de la dynamique des masses d'air et de leurs interactions susceptibles de déclencher des précipitations doit être faite à partir d'informations concernant des éléments qui se situent à 1000 ou $2000 \mathrm{~km}$ de la France et le plus souvent là où la densité d'observations est très faible. Les incertitudes qui en résultent, jointes aux inévitables approximations des schémas d'évolution des systèmes frontaux, n'autorisent pas une grande précision dans la localisation des précipitations maximales : or un examen de la zone affectée par les crues du 4 octobre 1960 montre qu'un déplacement de quelques dizaines de kilomètres du centre de gravité des pluies aurait considérablement modifié la physionomie des crues et la répartition des régions sinistrées.

Quant à la prévision quantitative des précipitations, si des considérations théoriques permettent de calculer a posteriori l'ordre de grandeur des phénomènes - et $M$. Fontaine a pu déduire, du soulèvement de la masse d'air chaud entre la la moyenne vallée de la Dordogne et le Plateau 
de Millevaches Ie 3 octobre 1960, une valeur de l'intensité des précipilations qui est recoupée à moins de $15 \%$ près par la valeur moyenne des intensités enregistrées par 7 pluviographes de la zone en question - sa mise en cenvre pratique suppose encore bien des progrès à réaliser en matière de prévision du temps, progrès que l'on peut d'ailleurs attendre de l'exploitation systématique de l'information météorologique existante, grâce à la puissance des moyens de calcul modernes.

Dans l'immédiat, on dispose des méthodes de prévision fondées sur les analogies de situations qui permettent, en procédant par approches de plus en plus précises au fur et à mesure que se dessine le contour du phénomène, d'apporter un concours efficace à l'annonce des crues. L'efficacité de ce concours est commandée par deux facteurs : d'une part l'établissement d'un échange continu d'informations entre les Services de la Météorologie et de l'Annonce des Crues tant que dure l'alerte qu'il s'agit de localiser progressivement, et d'autre part la rapidité de transmission et d'exploitation des informations échangées.

\section{B. - ANNONCE DES CRUES}

Ce problème peut être traité indépendamment de celui de la prévision, bien qu'il en constitue le prolongement logique : l'efficacité d'une prévision météorologique de crue n'étant réelle que si l'on sait passer des précipitations prévues à l'hydrogramme résultant à l'exutoire du bassin de réception. Il s'agit donc d'étudier la transformation pluie-débit dans la zone de formation de crue, les facteur's qui la conditionnent et ensuite la propagation de l'onde de crue ainsi constituée vers l'aval. Nous nous attacherons surtout ici à la phase de mise au point d'une méthode de calcul rapide de l'hydrogramme, laissant délibérément de côté la question de l'organisation pratique d'un service d'Annonce de Crues.

La mise au point de telles relations, étroitement subordonnées à la qualité des données expérimentales sur les précipitations et les débits, a pu être illustrée de façon très concrète à l'occasion du phénomène particulier qui nous occupe grâce à la convergence sur un objectif précis celui de la prévision des débits - des travaux effectués par diverș organismes.

1. Le Service Hydrométéorologique de la Production Hydraulique d'Electricité de France a mis en place un réseau de stations de mesures pluviométriques particulièrement dense sur les hauts bassins des rivières issues du Plateau de Millevaches, où l'on décompte, sur $8000 \mathrm{~km}^{2}$ environ, une centaine de pluviomètres simples et une vingtaine de pluviographes.

Ce réseau, dont l'équipement a été étudié en vue d'une sécurité de fonctionnement et d'une précision des mesures accrues, a permis de recueillir des renseignements précieux pour l'analyse fine de la situation météorologique à l'origine des précipitations des 3 et 4 octobre 1960 , cela grâce au tracé des isohyètes, des lignes isochrones de débul, des maximums et de fin d'averse, et à l'étude des intensités de pluie.

M. Guillot signale que ces intensités n'ont rien eu d'exceptionnel si l'on considère les intensités de pointe $(30 \mathrm{~mm} / \mathrm{h}$ pendant moins d'une heure autour de Millevaches le 3 en fin d'aprèsmidi) - surtout en comparaison de celles réalisées le 4 octobre 1958 dans le Gard (100 mm en $1 \mathrm{~h}$ à Canaules). Leur caraclère exceptionnel réside ici dans la durée (15 h) pendant laquelle une intensité moyenne voisine de $10 \mathrm{~mm} / \mathrm{h}$ a été observée sur plusieurs centaines de $\mathrm{km}^{2}$ des Monts des Monédières et du Plateau de Millevaches.

2. En regard de cette documentation pluviométrique, on dispose d'un outil d'étude remarquable avec les analyses de débits et d'hydrogrammes de crues effectuées par la $4^{\mathrm{e}}$ Circonscription Electrique en 16 points de mesures de la zone affectée par les crues. De la documentation hydrologique rassemblée dans le rapport de MM. Pellecuer et Barrière, nous extrairons les résultats suivants, définissant les traits généraux des crues du 4 octobre :

- La formation de ces crues a été favorisée par la forte hydranlicité des mois d'août et septembre 1960, qui atteint $200 \%$ sur les rivières issues du Plateau de Millevaches;

- Les limnigrammes des crues aux différentes stations présentent des vitesses moyennes de montée de 10 à $35 \mathrm{~cm} / \mathrm{h}$ (pour des temps de montée de 13 à $24 \mathrm{~h}$ ). Ces vitesses sont toutefois bien moins rapides que sur les torrents cévenols où l'on dépasse le $\mathrm{cm} / \mathrm{mn}$;

- Les débits spécifiques maximaux ayant dépassé $1 \mathrm{~m}^{3} / \mathrm{s} . \mathrm{km}^{2}$ ont été observés sur la Corrèze dont le bassin est couvert en entier par 
l'isohyète $140 \mathrm{~mm} \quad\left(1200 \mathrm{l} / \mathrm{s} . \mathrm{km}^{2}\right.$ à Tulle pour $370 \mathrm{~km}^{2}$ ) : le record étant détenu par la Vimbelle, affluent de rive droite débouchant à l'amont de Tulle, avec $1300 \mathrm{l} / \mathrm{s} . \mathrm{km}^{2}$ pour $140 \mathrm{~km}^{2}$. Par contre, la Vézère et la Vienne ont connu des maximums plus atténués: les barrages réservoirs de leurs cours amont se trouvant fortuitement en état d'encaisser des volumes assez importants de la crue.

- Ces débits spécifiques maximaux, relativement modestes comparés à ceux des rivières cévenoles, n'en ont pas moins constitué des records pour toutes les stations considérées: ils ont surclassé de loin les plus forts débits de crues connus.

-- On a pu vérifier que $72 \mathrm{~h}$ après le début de la crue, les cours d'eau touchés avaient évacué environ $50 \%$ de la lame d'eau reçue par leurs bassins respectifs les 3 et 4 octobre.

- Des considérations hydrogéologiques et géomorphologiques (nature des roches et des sols, pente des versants, hiérarchisation ou concentration du réseau hydrographique) ont permis d'expliquer les singularités de comportement de certains cours d'eau : étalement ou concentration des crues dans le temps.

- Enfin l'évolution des crues vers l'aval s'est traduite par une atténuation assez rapide, tant sur la Dordogne que sur la Vienne et le Cher, ainsi que l'observe M. Pardé dans une lettre très documentée adressée à la Commission : à Bergerac sur la Dordogne, à Châtellerault sur la Vienne, à La Roche-Posay sur la Creuse et à Noyer sur le Cher les maximums de 1960 n'ont pas atteint les records connus du passé.

Les évaluations de débit maximum au droit des stations par le Service des Débits de la $4^{\circ}$ Circonscription Electrique ayant fait l'objet de notes de calcul détaillées, des enseignements fort intéressants ont été dégagés sur la valeur relative des divers modes d'estimation indirecte des débits de crues et la nécessité de doubler les stations de jaugeage classiques d'une section auxiliaire judicieusement choisie pour l'évaluation des débits de crues.

3. La crue du 4 octobre 1960 a affecté l'un des bassins versants expérimentaux exploités par le Service des Etudes Hydrauliques d'E.D.F. en vue de la recherche de relations entre la pluie et l'écoulement. La station du Pont du Loubeix sur la Diège (B.V. : $225 \mathrm{~km}^{2}$ ) a connu le 4 octobre une crue de $730 \mathrm{l} / \mathrm{s} . \mathrm{km}^{2}$ étudiée par M. d'Harcourt. Il s'agissait de vérifier dans quelle mesure un hydrogramme unitaire, déduit de l'analyse d'une série de crues de moyenne importance, permettait de reconstituer l'hydrogramme d'une crue aussi exceptionnelle que celle-ci. Le résultat obtenu est intéressant: le temps de base et le temps de montée des hydrogrammes calculé et observé coïncident et la valeur du débit de pointe est restituée à $20 \%$ près. Ceci signifie que l'hydrogramme unitaire utilisé est une fonction de transfert acceptable pour définir la répartition dans le temps d'un volume de ruissellement correspondant à une averse de durée déterminée.

Dans le cas présent, le volume de ruissellement était équivalent à une lame d'eau de $27 \mathrm{~mm}$ fournie par une averse efficace de durée $16 \mathrm{~h}$ et d'intensité moyenne $8 \mathrm{~mm} / \mathrm{h}$. Le coefficient de ruissellement de $20 \%$ relatif à une telle crue peut paraître faible : il est lié d'une part à la faiblesse des intensités de pluie et d'autre part à la nature de la couverture végétale favorisant l'infiltration.

Toutefois la portée pratique de cet essai de relation pluie-débit est limitée, car la reconstitution ne porte que sur l'hydrogramme de ruissellement, non sur l'hydrogramme d'écoulement total (ruissellement + écoulement souterrain), et de plus c'est le volume de ruissellement déduit de la crue observée qui est ici modulé dans le temps par l'hydrogramme unitaire. Deux problèmes essentiels demeurent donc posés:

- déterminer la fraction d'averse qui produit le ruissellement: étude des coefficients de ruissellement d'averses en fonction de paramètres caractérisant l'état du bassin;

- déterminer les variations dans le temps des diverses phases d'écoulement constitutives de l'hydrogramme global: variation de la capacité d'infiltration $\mathrm{du}$ bassin en fonction de la durée de l'averse, lois d'évolution de la crue et de la décrue d'écoulement souterrain.

4. La méthode de calcul d'hydrogrammes à partir des pluies proposée par M: Lacroix apporte au problème de l'annonce des crues une solution séduisante, en ce sens qu'elle permet de déterminer point par point l'hydrogramme de crue global, d̀ des intervalles de temps aussi rapprochés que l'on veut, au fur et à mesure des informations reçues concernant les précipitations tombées sur le bassin. L'efficacité du procédé tient d'une part à sa rapiditè de mise en œuvre (simples lectures d'abaques et reports graphiques) et d'autre part à ses possibilités d'application $\dot{a}$ des bassins versants de grandes dimensions, alors que la méthode de l'hydrogramme unitaire sous sa forme classique n'est plus utilisable en général lorsque le bassin versant dépasse quelques centaines de $\mathrm{km}^{2}$.

M. Lacroix part des principes suivants :

- La pluie réelle tombée à chaque instant sur un bassin élémentaire se répartit en deux phases: l'une qui ruisselle, l'autre qui s'in- 
filtre. La première alimente une composante du débit correspondant à un écoulement relativement rapide $Q_{r}$ (avec un décalage de temps de l'ordre de quelques heures sur un bassin de 500 à $1000 \mathrm{~km}^{2}$ ) et la seconde, une composante d'écoulement relativement lent $Q_{i}$ (décalé de un ou plusieurs jours); le débit réel à l'exutoire du bassin étant constitué par la somme de ces deux composantes;

-- On définit les transformations permettant de passer des pluies réelles aux débits qu'elles produisent, compte tenu des divers décalages de temps :

$1^{\circ}$ La répartition de la pluie réelle entre les deux phases « ruissellement » et infiltration 》 est faite d'après un coefficient caractérisant la fraction $d u$ bassin qui donne lieu à ruissellement;

$2^{\circ}$ Le passage du volume de pluie d'une des phases ainsi définies au volume d'écoulement qu'elle produit (passage d'une pluie réelle à la pluie nette) fait intervenir les pertes ou déficits d'écoulement. La transformation de réduction que l'on fait subir à la pluie est fonction de deux paramètres : le débit d'écoulement lent $Q_{l}$, qui caractérise l'état du bassin, et le montant de la pluie à transformer. On définit ainsi deux transformations de réduction qui s'appliquent aux deux phases d'écoulement considérées;

$3^{\circ}$ Disposant alors des valeurs des volumes d'écoulement - « rapide » et «lent» - correspondant aux pluies nettes, on définit des transformations d'étalement qui conservent ces volumes et donnent leur distribution dans le temps. Ainsi, à ce stade, $M$. Lacroix s'est attaché $\dot{a}$ résoudre de façon pratique le problème de la définition des pluies nettes auquel achoppent toutes les tentatives d'utilisation systématique d'hydrogrammes unitaires;

$4^{\circ}$ Les transformations d'étalement E qu'il propose ensuite de substituer à l'hydrogramme unitaire sont définies comme des transforma- tions de réservoirs où le débit sortant $Q_{s}$ dépend uniquement $d u$ volume stocké $V$ : la variation du volume est liée au débit par une fonction $\mathrm{T}$, homogène à un temps, qui représente la rapidité de vidange du stock par le débit $Q_{s}$. Cette fonction étant définie expérimentalement d'après les hydrogrammes de crue observés, on dispose d'une transformation spécifique pour chacun des types d'écoulements;

$5^{\circ}$ L'application de ces transformations aux volumes d'écoulement correspondants permet de définir l'hydrogramme de cruc relatif à la zone élémentaire considérée.

Pour tenir compte de la propagation de la crue dans le cours d'eau principal et obtenir ainsi une meilleure chronologie du phénomène (les temps de propagation diminuent fortement lors des très grandes crues, du type de celles d'octobre 1960), M. Lacroix propose de découper le bassin versant en zones élémentaires de l'amont vers l'aval, la combinaison des hydrogrammes relatifs à chaque zone s'effectuant avec đes décalages de temps qui sont fonction des débits transitant dans le cours d'eau principal.

La mise au point de ces diverses iransformations a été faite empiriquement par des procédés graphiques à partir d'une série de hyélogrammes et d'hydrogrammes de crues de la Corrèze à Brive $\left(1000 \mathrm{~km}^{2}\right)$, bassin qui se prêtait bien à une telle recherche, étant donné l'absence d'aménagements hydroélectriques importants sur son cours et son homogénéité géologique.

Cette méthode de calcul d'hydrogrammes paraît constituer, quand on considère le nombre et la variété des cas de concordance d'hydrogrammes présentés par M. Lacroix, une voie pleine de promesses pour la prévision des débits à partir de la pluie. A cet égard, il serait très souhaitable qu'elle soit expérimentée sur d'autres bassins versants et fasse l'objet de travaux concertés de la part des hydrologues qui s'intéressent tant à l'annonce des crues qu'au problème plus général de la prévision des débits.

\section{C. - PROTECTION CONTRE LES CRUES}

L'annonce des crues ayant pour but essentiel d'assurer la protection des populations et des biens dans les zones où les submersions sont inévitables, il reste à déterminer ces zones critiques où des travaux de défense pourront être effectués et à définir les aménagements à réaliser en fonction des risques à couvrir. Les observations et analyses effectuées à l'occasion des crues du 4 octobre 1960 ont fourni à ce sujet une ample moisson d'informations ayant trait d'une part à la localisation de l'activité géomorphologique des cours d'eau de l'ouest du Massif Central et d'autre part, à l'estimation de la durée de retour de phénomènes d'une telle importance.

1. L'examen systématique des indices d'activité géomorphologiques des rivières, auquel se sont livrés MM. Hlavek et Lecarpentier, a permis de classer les dégâts observés en «dégâts naturels», liés à la dynamique propre à chaque 
cours d'eau et à son évolution normale, et en «dégâts anthropiques »ui résultent d'une inadaptation du lit et des ouvrages qui l'enjambent ou le bordent du fait de l'intervention humaine.

L'activité géomorphologique a été dans l'ensemble assez faible: pas de transports solides, débordements sans action morphologique importante sauf dans les sinuosités (érosions, atterrissements du fait des recoupements de méandres). Parmi les causes de cette modération, il faut ranger le relief peu accentué de la zone affectée par la crue, son réseau hydrographique hiérarchisé, sa couverture végétale à peu près totale, la relative faiblesse des intensités de précipitations (il apparaît ainsi que le ruissellement de surface n'a été ni généralisé, ni abondant) et enfin l'existence de retenues de barrages dont l'effet d'écrêtement et d'étalement sur les crues a été sensible.

Les dégâts «anthropiques》 ont été surtout localisé aux ponts et sur les chaussées d'accès en remblais : ponts mis en charge ou contournés, affouillements de piles ou de culées dans le cas de ponts établis dans une courbe ou à l'aval immédiat d'une sinuosité. Les biefs des moulins, les endiguements latéraux en courbe et principalement les empiètements des constructions sur le lit mineur dans la traversée des localités, les rétrécissements de lit (Tulle) ont été cause de dégâts importants.

Par-delà ces constatations sur la nature des dégâts et leurs causes, il apparaît que, soumis à des précipitations comparables, les divers cours d'eau étudiés ont réagi assez diversement et que les différences constatées sont liées à la nature des bassins versants. Ce qui permet à MM. Hlavek et Lecarpentier de distinguer, d'après des critères d'activité géomorphologiques, divers types de secteurs ou zones critiques et de définir les aménagements susceptibles d'assurer leur protection.

2. Les coûts de tels aménagements d'ensemble d'un bassin versant (traitement des versants, création de réservoirs) ou de détail (rectification de cours, digues de protection, déversoirs, etc.), doivent être d'abord mis en balance avec le coût des dégâts en cas d'insuffisance de protection : la décision du maître d'œuvre résultera ensuite de la confrontation de ces coûts et des probabilités des phénomènes dont on désire se protéger. Il était donc intéressant d'examiner la durée de retour d'un évènement aussi excep- tionnel que celui du 4 octobre 1960 sur le nordouest du Massif Central.

MM. Pellecuer, Guillot et d'Harcourt ont pour cela étudié les distributions des valeurs maximales des débits journaliers et des relevés pluviométriques de 24 ou 48 h. Dans l'un et l'autre cas, la méthode utilisée a été l'ajustement d'une loi de Valeurs Extrêmes (Gumbel ou Fréchet) à un échantillon de valeurs maximales annuelles de la variable considérée.

Les résultats ont été, comme il fallait s'y attendre, très dispersés, étant donné qu'il n'existe pas de critère objectif de choix a priori pour le type de loi de probabilité à utiliser. M. Pellecuer a pu montrer, à propos de l'étude des débits de la Corrèze à Brive, que les ajustements avec une loi de Gumbel et une loi de Fréchet étaient également corrects : la durée de retour de la crue de 1960 passant de 1000 à 97 ans...

De plus la prise en compte de l'année 1960 dans l'échantillon utilisé pour l'ajustement de la loi de Valeurs Extrêmes se traduit par un relèvement sensible de la courbe expérimentale. Comme il n'y a aucune raison a priori d'exclure cette année de la période d'observation et que, d'autre part, la fréquence expérimentale attribuée à la crue de 1960 est certainement trop forte en raison du petit nombre d'années d'observations continues dont on dispose (de 30 à 50 ans en moyenne), il paraît sage de s'en tenir à la partie commune des intervalles de confiance à $70 \%$ associés aux droites de Fréchet ou Gumbel, calculées à partir des observations comprenant ou excluant l'année 1960, pour définir la zone probable correspondant à la durée de retour du phénomène. Dans ces conditions on peut raisonnablement estimer avec $M$. Pellecuer la durée de retour de la crue d'octobre 1960 à une valeur comprise ente 100 et 200 ans.

Une question mériterait d'ailleurs d'être soulevée à ce propos : peut-on penser que la crue de 1960 fait partie de la même «population», au sens statistique, que les crues maximales annuelles des années antérieures? Autrement dit, l'échantillon de valeurs extrêmes comprenant l'année 1960 est-il homogène? On pourrait en douter si l'on se réfère au caractère «hors série» mis en évidence par l'analyse de la situation météorologique que M. Fontaine a présentée. C'est dire avec quelle prudence il faut faire état des «durées de retour 》 de tels phénomènes dont la répartition dans le temps est par ailleurs inconnue. 


\section{CONCLUSION}

Parler de « conclusion » risque de faire entendre que l'hydrométéorologie des crues de cette région du Massif Central ne pose plus de problèmes... Il s'agit plutôt, après l'exposé des travaux. qu'elles ont suscités, de dégager les orientations de recherches qui en découlent.

En reprenant les têtes de chapitres d'étude des crues : Prévision, Annonce, Protection, on peut dresser rapidement une liste de sujets sur lesquels il paraît utile — et rentable du point de vue de l'efficacité du contrôle de ces phénomènes - de promouvoir des recherches ou de procéder à des mises au point. Cette liste n'est èvidemment pas exhaustive et certaines propositions débordent peut-être le domaine des travaux de la Commission pour l'Etude des Débits de crues, tant il est inévitable que des interférences de compétences se produisent dans l'étude des phénomènes naturels.

\section{Prévision}

Il serait banal de répéter ici que tous les progrès en matière de prévision quantitative des précipitations sont d'une importance primordiale. Deux points d'application des efforts dans cette voie paraissent susceptibles d'apporter rapidement une contribution efficace à la prévision des crues :

- La recherche et l'analyse des situationstypes génératrices de crues sur les différents bassins fluviaux effectuées dans la ligne des travaux de M. Fontaine (crues de 1955 sur la Seine, de 1958 sur les rivières cévenoles, de 1960 sur les rivières issues du Plateau de Millevaches). Cette définition de situations météorologiques-types, permettant de distinguer des catégories de crues d'un même cours d'eau constitue par ailleurs la préface nécessaire aux études d'hydrologie statistique sur la répartition des crues dans le temps et par importance;

- Un examen approfondi des possibilités d'utilisation des radars météorologiques à la localisation des maximums pluviométriques devrait être entrepris en ce qui concerne particulièrement l'interprétation des échos correspondant à divers types de précipitations.

\section{Annonce}

L'annonce des crues étant étroitement conditionnée par des impératifs de rapidité et de sécurité de fonctionnement des dispositifs de télémesures pluviométriques et limnimétriques et de transmission des observations, on dispose dans ce domaine d'une gamme de réalisations éprouvées (compteurs de pluie, téléindicateurs de niveau, «pluviophones», «limniphones», etc.) dont on peut envisager à l'heure actuelle l'installation dans les zones de formation de crues.

En ce qui concerne l'hydrométrie, la confrontation des méthodes de mesures directes et d'estimation indirecte des débits de crues devrait être l'occasion d'une mise au point utile orientée vers le choix et l'aménagement des sections de mesure.

L'analyse d'un système d'annonce de crues en vue de son amélioration revient à chercher quelles solutions il apporte aux deux problèmes de transformation de la pluie en débit et de propagation d'ondes de crues dans un réseau hydrographique complexe :

- A ce sujet, s'il s'avère que les promesses de la méthode indiquée par M. Lacroix pour le calcul des hydrogrammes à partir de la pluie, se vérifient dans des applications à divers types de bassin et à une grande variété de crues, son intérêt serait d'autant plus grand qu'elle paraît susceptible d'une programmation simple sur calculateur électronique.

- Quant à la propagation des crues, s'il existe d'assez nombreux travaux la concernant, un état de la question et une synthèse des résultats acquis restent à faire.

\section{Protection}

Dans ce domaine, un premier sujet de réflexion apparaît dès qu'il s'agit de définir les «zones critiques» à protéger. Des critères d'ordre humain et économique (densité de population dans les zones inondables, coût des dégâts, etc.) doivent être conjugués à des analyses géomorphologiques dont l'intérêt n'est plus à démontrer lorsqu'il s'agit d'expliquer les différen- 
ces de comportements de bassins versants soumis aux mêmes conditions météorologiques.

Enfin l'aspect probabiliste de l'étude des crues, que l'on retrouve dans tous les problèmes de protection contre leurs effets, rappelle que bien des choses restent à faire, ou plus exactement que l'on ne sait pas grand'chose sur la probabilité des phénoménes «exceptionnels »... En 1959, M. Morlat présentait ici même une esquisse de programme pour les travaux ultérieurs de la Commission où figurait la question des probabi- lités météorologiques : le moins que l'on puisse dire est qu'elle y demeure...

$$
\text { *** }
$$

Cette énumération - non limitative - - montre que les futurs travaux de la Commission ne manqueront pas de matière, d'autant qu'il s'agit ici uniquement des crues d'origine pluviale: l'étude des mécanismes de formation des crues de fonte des neiges devra être envisagée à part.

\section{I S C US S I O N}

Président: M. de Rouville

Après l'exposé de M. JacQuet, M. le Président félicite l'auteur pour son travail, préparé en relativement peu de temps et exposé dans les limites imparties.

M. le Président pense que de telles séances d'études ne sont pas trop nombreuses : une étude semblable sur les crues de 1930 dans l'Aveyron, le Lot et le Tarn n'aurait pas été inutile et les crues à l'étranger peuvent être aussi très instructives.

\section{$*$}

Après les commentaires apportés par les auteurs des communications présentées à la Commission le 5 mai 1961, M. JACQUET donne alors lecture de ses conclusions qui sont adoptées, comme celles de la Commission, par le Comité technique.

M. Remenieras précise la recommandation concernant, pour l'ensemble du territoire, la délimitation des «zones critiques $\gg$ au point de vue des inondations.

Dans les zones ainsi délimitées, d'une part la construction d'immeubles serait interdite, en principe, ce qui éviterait à l'Etat et à la communauté de payer des dédommagements pour les dégâts provoqués par les inondations, d'autre part, il serait procédé à des études spéciales (bassins versants expérimentaux, notamment) en vue de préciser les limites des zones inondées par les crues de diverses fréquences et d'étudier la rentabilité d'ouvrages de protection.

M. le Président croit aussi que le Comité pourrait aller plus Ioin que la simple étude des crues dans le centre de la France et énumère les zones critiques d'Alsace et du Nord, où les vallées sont très plates et où les rivières débordent facilement.

Le Bureau de la S.H.F. pourrait alors porter ces conclusions à la connaissance des services intéressés : Service de la Décentralisation du Territoire ou Service de la Protection Givile. M. le Président insiste aussi sur le fait que les organisations de protection, matériel et ouvrages, doivent être vérifiées pour qu'on soit sûr qu'elles fonctionneront au moment voulu.

M. Ie Président clôt cette partie de séance en souhaitant que les observations apportées permettent de progresser et d'éviter les pires conséquences.

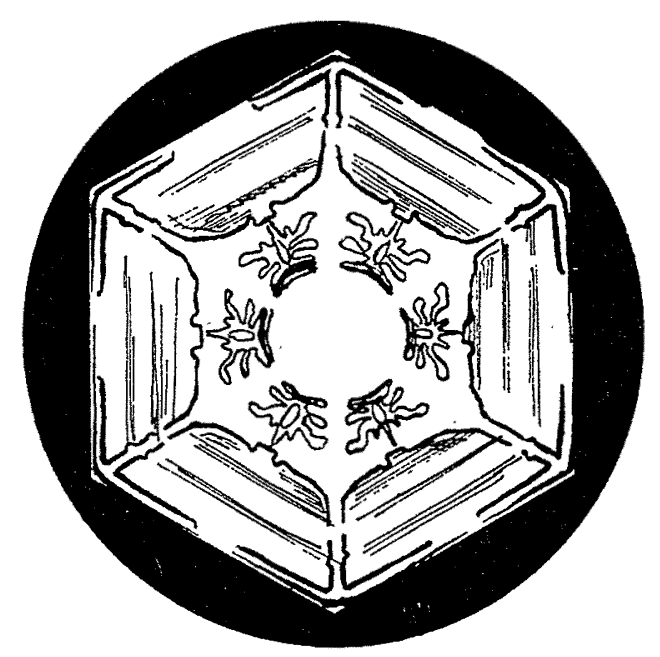

